\title{
ORIENTARSE EN INTERNET: HYTELNET 6.4
}

\section{Ortiz-Repiso Jiménez y C. Olmeda Gómez *}

Resumen: Se describe la versión 6.4 del programa Hytelnet, diseñado por Peter Scott. Este programa es una herramienta muy útil para los usuarios de Internet, ya que permite una orientación y un fácil acceso a los recursos que la red ofrece. Hytelnet es un programa residente en memoria con características de hipertexto de fácil aprendizaje, uso e instalación. Contiene más de un millar de direcciones telnet. Se describe su manejo al mismo tiempo que se explica el tipo de información que contiene. Se proporciona el modo de obtener hytelnet para diferentes sistemas operativos, así como las actualizaciones que periódicamente se realizan.

Palabras clave: Hytelnet, Internet, redes informáticas.

\begin{abstract}
The Hytelnet programme version 6.4, designed by Peter Scott, is described. This programme is a highly useful tool for the Internet user, as it can both orient and give easy access to the network's resources. The programme contains more than a thousand telnet addresses. Hytelnet is a terminate and stay resident programme in the computer's memory, with hypertext features which are user friendly in terms of both learning and installing. It describes how to use the programme and the information it contains at the same time. Finally, the article gives details on how to incorporate Hytelnet into different operating systems, as well as the regular updates that are made.
\end{abstract}

Keywords: Hytelnet, Internet, Networks.

\section{Introducción}

Como es conocido por todos, Internet es una gran red de redes, de ámbito universitario y de investigación, en la que se calcula que están interconectadas más de dos mil redes. Es difícil estimar el número de ordenadores conectados en el conjunto de redes, y se piensa que el número de usuarios diarios que acceden a Internet es de tres millones, distribuidos por América, Europa, Asia, Africa y Australia. Estas cifras aumentan día a día, y se cree que el número de usuarios que se conectan crece a un ritmo del $10 \%$ mensual.

Esta red nos ofrece una infraestructura sin precedentes para compartir recursos. Un usuario de Internet puede conectarse a un ordenador en la otra parte del mundo, tan rápida y fácilmente como si estuviera en la habitación contigua.

Cuando un usuario comienza a utilizar Internet, uno de los mayores problemas con los que se encuentra es la gran cantidad de información y servicios que existen en la red. En el mundo bibliotecario estamos más acostumbrados a oír hablar de catálogos de bibliotecas disponibles para su consulta vía Internet, pero esto es sólo una parte de lo que la red ofrece. Internet incluye servicios de acceso a bases de

* Universidad Carlos III de Madrid. Departamento de Biblioteconomía y Documentación.

Recibido 20-8-93. 
datos comerciales, bibliografias, correo electrónico, revistas electrónicas, transferencia de ficheros, acceso a servidores (Archie, Gopher...) y un largo etcétera. Ahora bien, descubrir, identificar y localizar los recursos de información en Internet no siempre es tarea fácil para aquellas personas que se inician en el uso de la red. Navegar por Internet para localizar y utilizar los servicios que nos ofrece es un verdadero desafio para los usuarios. Esto se debe, principalmente, a dos motivos: Internet no tiene un único administrador sino que cada red interconectada tiene el suyo propio; la red está en continuo crecimiento y los cambios son frecuentes.

Los usuarios necesitan saber qué sistemas son accesibles en la red, encontrar qué recursos de información están disponibles en cada sistema, usar los comandos telnet para acceder a los sistemas deseados, conocer su login, saber buscar en ellos y saber salir de los mismos para volver a su propio sistema local.

Hasta hace poco las únicas herramientas disponibles para ayudar a los usuarios de la red a conectarse a los catálogos en línea de acceso público (en adelante OPACs) y a otros sistemas de información, eran tres grandes directorios: la guía de recursos de Internet (1) (compilada por el National Science Foundation Network Service Center), el directorio de Art. St. George (2) y el directorio de Billy Barron (3). Todos ellos proporcionan direcciones telnet e instrucciones de acceso. Pueden obtenerse vía FTP (anonymous) y almacenarlos en un sistema local.

Desde 1990 se han desarrollado varios programas con versiones en DOS, Macintosh y UNIX/VMS, que tomando como base la información de los directorios citados, especialmente los dos últimos, constituyen herramientas muy útiles para aprovechar y explotar al máximo los recursos de Internet, tanto para el usuario que se inicia como para aquellos más expertos, ya que permite acceder a las direcciones telnet de una manera fácil, rápida, cómoda y actualizada.

Este articulo habla de uno de estos programas, Hytelnet, en la versión 6.4 para DOS, de 1 de febrero de 1993.

\section{2 ¿Qué es Hytelnet?}

Hytelnet es un programa diseñado por Peter Scott, del Departamento de Sistemas de Bibliotecas de la Universidad de Saskatchewan de Canadá. Hytelnet contiene más de un millar de direcciones de los diferentes servicios accesibles vía telnet.

Este programa se utiliza para ayudar a localizar las direcciones vía telnet de:

- Catálogos de acceso público en línea (OPACs).

- Bases de datos y biblio grafías.

- Servicios de correo electrónico.

- Boletines electrónicos de noticias.

- Revistas electrónicas.

- Servicios de información general de universidades.

- Libros en formato electrónico.

- Servidores (Archie, Gopher, WAIS, WWW).

- Servicios directorio y páginas amarillas. 
- Acceso a otras redes.

- Cualquier otro servicio accesible en Internet.

Hytelnet recoge y amplía la información contenida en los directorios de Art St. George y Billy Barron. El primero de ellos incluia inicialmente sólo direcciones de OPACs accesibles en Internet. Posteriormente, la obra se amplió con direcciones de servicios de información general de universidades, boletines electrónicos de noticias, asi como direcciones de revistas electrónicas. Divide la sección de OPACs en tres apartados: uno para los de libre acceso; otro para los que son accesibles previo pago; y un tercero para los OPACs de bibliotecas que no pertenecen a los Estados Unidos. Todos ellos están ordenados por estado, provincia o país. Incluye, también, información sobre los procedimientos básicos de conexión (algunas veces muestra una pantalla con los símbolos del sistema), la persona de contacto y, en ocasiones, una descripción del contenido del catálogo.

El directorio de Barron complementa la obra de St. George, ya que proporciona un formato normalizado para las direcciones Internet de todos los sistemas recogidos en la obra: dirección Internet, instrucciones para la conexión y la desconexión y nombre del sistema de automatización que usa la biblioteca. En un apéndice recoge los nombres comerciales de los programas de las bibliotecas, instrucciones básicas para el uso de los OPACs y persona de contacto con su dirección.

\section{Características de Hytelnet versión 6.4}

Tamaño del programa: 16.575 bytes (fichero Hr.exe).

Tamaño del programa instalado: 62.313 bytes (67 ficheros).

Tamaño del fichero ASCII: máximo de $24 \mathrm{~Kb}$.

Existen versiones del programa para IBM y ordenadores compatibles que trabajen con DOS, para Macintosh y para UNIX/VMS.

Hytelnet es un programa residente en memoria con características de hipertexto. $\mathrm{Su}$ autor lo define como un hypertext browser (4) que permite al usuario conseguir un acceso instantáneo a direcciones accesibles por telnet.

El ser un programa residente en memoria facilita su consulta, ya que puede activarse en cualquier momento de una sesión de trabajo en Internet. Aunque no inicia o realiza la conexión (en las versiones en PCs) es un sustituto muy útil de los directorios citados anteriormente. Hytelnet debe cargarse en la memoria antes de iniciar el programa de comunicaciones. Una vez cargado y estando conectados a Internet, si necesitamos una dirección telnet, sólo tenemos que activar el programa con la combinación Ctrl. + Backspace (simultáneamente). Si queremos abandonar momentáneamente el programa pulsamos Esc., pudiendo reactivarlo del mismo modo ( $\mathrm{Ctrl}$. + Backspace) cuantas veces sea preciso. Si deseamos retirarlo de la memoria se pulsa la combinación Alt $+T$.

La información que contiene Hytelnet puede personalizarse, modificando un archivo ya existente o añadiendo uno nuevo que contenga, por ejemplo, una nueva dirección telnet. Los ficheros podemos editarlos con cualquier tratamiento de textos, con la única precaución de archivarlos en formato ASCII. Hytelnet facilita información de ayuda para realizar estas tareas en el fichero $<$ WHATIS $>$. 
Hytelnet está construido con el software de hipertexto (HyperRez) propiedad de Neil Larson, producido por la empresa Maxthink especializada en elaborar aplicaciones de este tipo. El hipertexto está diseñado para que recuerde un máximo de 64 saltos, lo que nos permite movernos o navegar por el programa con gran agilidad.

\section{4 ¿Qué contiene Hytelnet?}

En el menú principal de bienvenido al sistema, figura 1, aparecen nueve ficheros. Tres de ellos están relacionados con información sobre bibliotecas; uno con otros recursos accesibles en Internet; y el resto son guías de ayuda para el usuario de Hytelnet y de Internet.

Figura 1

Menú principal: Bienvenido a Hytelnet

Welcome to HYTELMET

version 6.4

What is HYTELNET?

रमHกT'IS

Library catalogs

〈SITES1>

Other resources

〈SITESZ〉

Help files for catalogs (OPBBB)

Catalog interfaces

〈SYSBBB〉

Internet Glossary

〈GLOSSARY〉

Telnet tips

〈TELNET)

Telnet/TH32P0 escape keys (ESCAPE. KEY)

Key-stroke commands (HELP. TXT)

\subsection{Información sobre bibliotecas}

El número total de bibliotecas cuyas direcciones telnet se incluyen en el programa y su distribución geográfica aparecen reflejados en la Tabla I.

Los ficheros del menú principal relacionados con bibliotecas son tres: Library catalogs $<$ SITES1 $>$, Help files for catalogs $<\mathrm{OP} 000\rangle$ y Catalog interfaces $<$ SYSO00 $>$. Estos ficheros están unidos entre si, pudiendo acceder a ellos por rutas diferentes. 
Tabla I

Número de bibliotecas y distribución geográfica

\begin{tabular}{|c|c|c|}
\hline Bibliotecias & N." OPACS & $\%$ \\
\hline TOTAL AMERICA & 403 & 65,5 \\
\hline Estados Unidos & 359 & \\
\hline Bibliotecas de colleges & 11 & \\
\hline Bibliotecas juridicas & 13 & \\
\hline Bibliotecas médicas & 23 & \\
\hline Otras bibliotecas & 312 & \\
\hline Canadá & 42 & \\
\hline México & 2 & \\
\hline TOTAL EUROPA & 163 & 26,5 \\
\hline Alemania & 7 & \\
\hline Austria & 2 & \\
\hline Bélgica & 2 & \\
\hline Dinamarca & 11 & \\
\hline España & $5^{1}$ & \\
\hline Finlandia & 11 & \\
\hline Francia & 1 & \\
\hline Holanda & 5 & \\
\hline Irlanda & 4 & \\
\hline Islandia & 1 & \\
\hline Israel & 7 & \\
\hline Italia & 1 & \\
\hline Noruega & $11^{2}$ & \\
\hline Reino Unido & 79 & \\
\hline Suecia & 10 & \\
\hline Suiza & 6 & \\
\hline TOTAL AFRICA & 1 & 0.1 \\
\hline TOTAL AUSTRALIA & 40 & 6,5 \\
\hline Australia & 35 & \\
\hline Nueva Zelanda & 5 & \\
\hline TOTAL ASIA & 8 & 1,4 \\
\hline Hong Kong & 3 & \\
\hline Taiwan & 5 & \\
\hline TOTAL MUNDIAL & 615 & 100 \\
\hline
\end{tabular}

I Se han contabilizado individualmente cada una de las direcciones recogidas en Hytelnet, pese a que las direcciones del CSIC-Madrid y CSIC-Sevilla dan acceso a diferentes bibliotecas, pero su numero no se indica en Hytelnet. En la versión 6.6 de 10 de octubre de 1993 se recogen 11 direcciones de OPACs españoles.

2 Pertenecientes a la Red BIBSYS de bibliotecas de investigación. 


\subsubsection{Catálogos de bibliotecas <SITES1 >}

Contiene direcciones IP de OPACs, ordenadas por continentes; en el ejemplo hemos seleccionado Europa/Escandinavia (Figura 2); y por paises, en el ejemplo Reino Unido (Figura 3).

Figura 2

Catálogos de bibliotecas

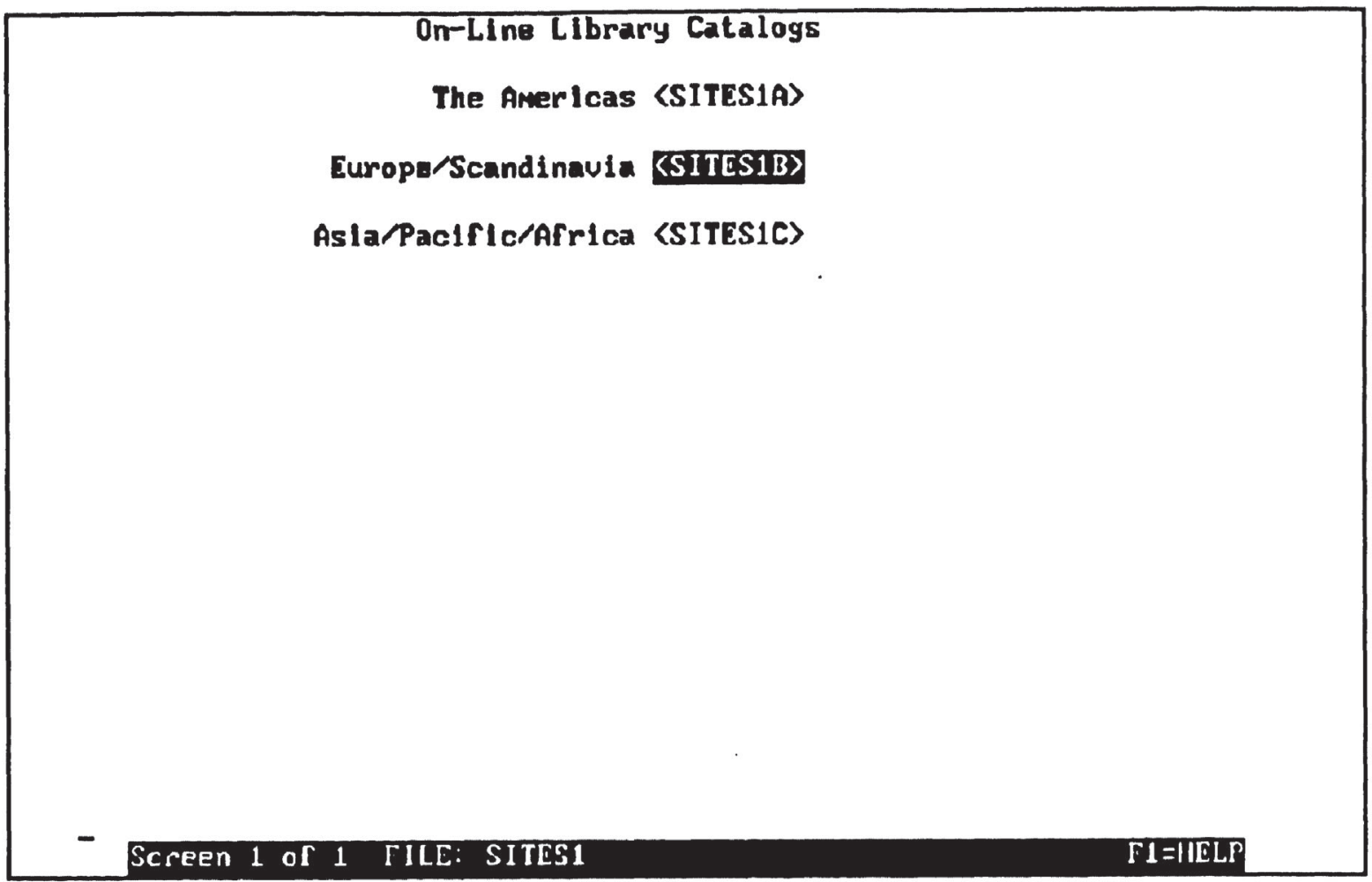

Al seleccionar el deseado aparecerá el listado de las bibliotecas de ese pais (Figura 4).

Una vez localizado un centro, situamos el cursor sobre la biblioteca elegida y accedemos a la dirección telnet (Figura 5).

Cada biblioteca, como vemos en el caso de la Biblioteca Nacional de Escocia, tiene su propio fichero que contiene la dirección telnet, el login a utilizar, el nombre del host, los comandos necesarios para conectarse y desconectarse, el tipo de terminal y el nombre comercial del OPAC que posee.

La versión 6.4 de Hytelnet permite desde esta misma pantalla acceder a las que describen las ayudas del OPAC. Estas pantallas nos ofrecen una pequeña información de cómo formular las búsquedas en cada uno de los distintos OPACs (Figura 6). 
Figura 3

OPACs en Europa y Escandinavia

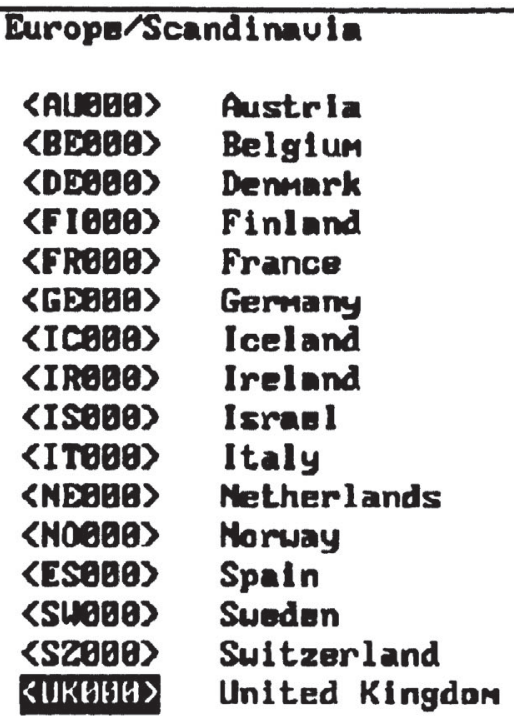

\section{Figura 4}

OPACs en el Reino Unido

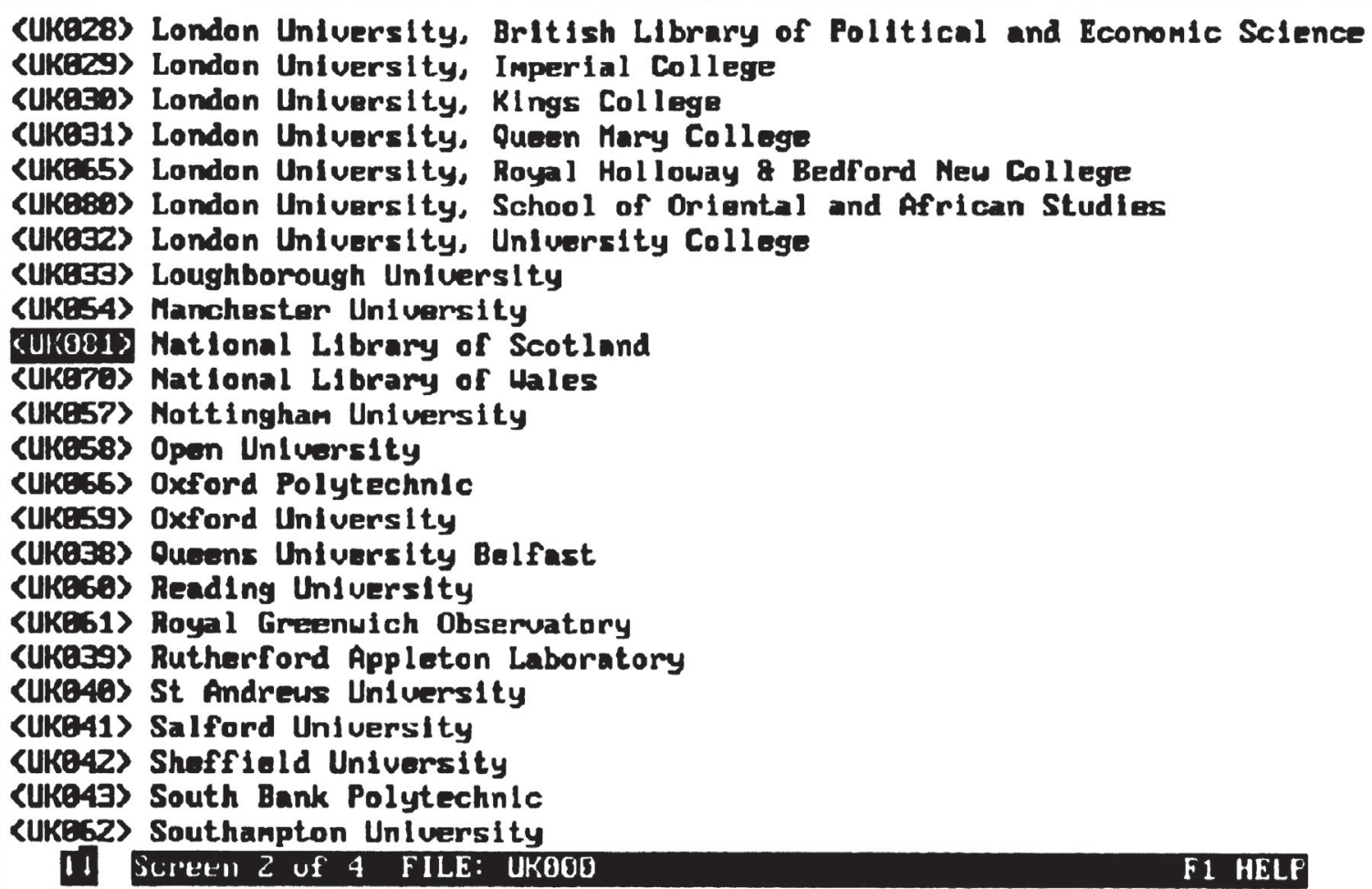


Figura 5

Biblioteca Nacional de Escocia

\section{Hatlonal Library of Scotland}

TELNET SUN. MSP. AC. UK or 128.86.8.7

login: janot

hostname: uk.nls.opac

MPE XL: hello username, ref.clas50

OPAC = UTLS EOPHI\%

To exit, type 0 on maln menu

Figura 6

Guía del OPAC de VTLS

Using UILS

Aduanced User Search System:

Author searches: To search for a particular author, use the Af search command followed by the author"s name.

Example: A/Heningway, Ernest

I1t le searches: To search for a particular title, use the $T$ search command followed by the title. Onlt any leading articles such as THE, $A, A N$, LA, ...

Example: I/Sun also rises

Subject searches: To search for a particular subject, use the so search command followed by the subject.

Examp 18: SHetals

Keyword searches: To search for a keyword, use the th search command followed by the subjoct.

Example: W/Computers

I1) SCIEEII 1 of 2 FILE: UP81? F1 HELP 


\subsubsection{Interfaces de los catálogos <SYSO00> y ficheros de ayuda para OPACs $<O P O 00>$}

Otra característica sobresaliente del programa es la posibilidad de ver qué bibliotecas utilizan un determinado OPAC. Se accede a través del fichero Catalog interfaces < SYSO00 > que contiene un listado de los diferentes sistemas de bibliotecas en uso (Figura 7).

Si nos situamos en VTLS, por ejemplo, aparece una nueva pantalla con todos aquellos centros que utilizan este sistema (Figura 8).

Desde aquí podemos elegir la biblioteca que deseemos, acceder a su dirección y desde ésta a la guía de utilización del OPAC (Figuras 6 y 7). Esta información es muy útil para los usuarios que se ven obligados a enfrentarse con interfaces muy variados. Con el ejemplo presentando podemos apreciar claramente, como antes señalábamos, la conexión que existe entre los diferentes ficheros del programa.

\section{Figura 7}

\section{Lista de OPACs}

\section{Library Systems currently in use}

〈SYSBQ1〉 DRA Altas sites

(SYSBO2) BLCMP Sites

〈SYSBO3〉 BuCAT sites

〈SYSBe4) CATS sites

(SYSEZZ) CLSI sites

〈SYSEZ1) DOBIS/LIBIS sites

〈SYSBo5) Dynix sites

(SYSBO6) GEAC sltes

(SYSO16) GEAC Aduance sites

〈SYSQ19〉 GuB sites

〈SYSO17) Inlex sites

〈SYSEOP) ImuOPAC sites

〈SYSBO8) Libertas sites

(SYSO18) LS/2000 sites

〈suSeze) Mult1lis sites

〈SYSBO9〉 MOTIS sites

(SYSEZ3) OCLC sites

〈SYSE1日) PALS sites

〈SYSO11) UNICORN sites

〈SYSO12〉 URICA sites

〈SYSO13) UTCAT sites

KSYSH11) UTLS sites

II SCreEll 1 of 1 FILE: SYSOVO F1 HELP 
Figura 8

Bibliotecas que usan VTLS

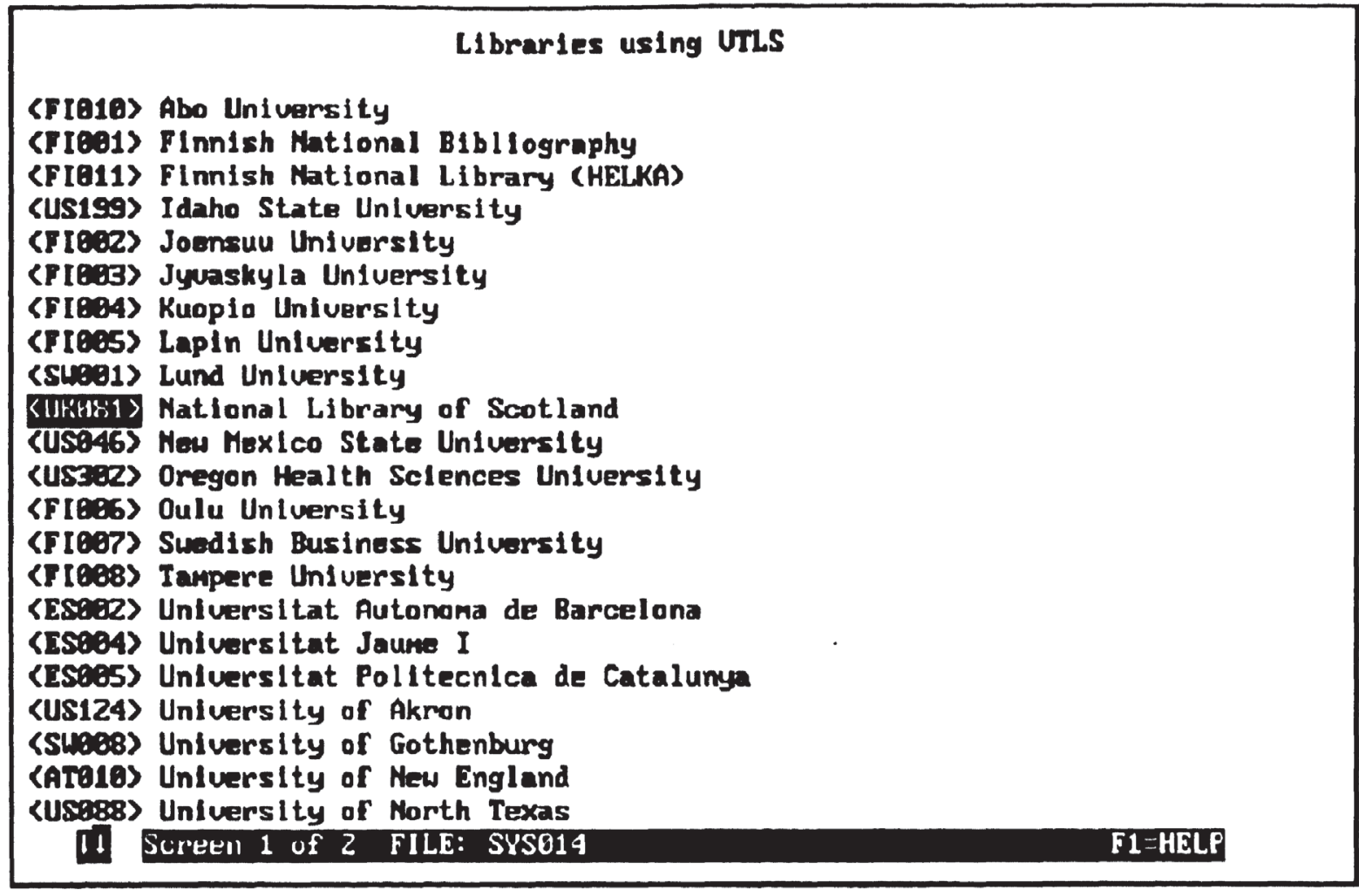

\subsection{Acceso a otros recursos $<$ SITES2 $>$}

Además de los OPACs, Hytelnet presenta otros recursos accesibles en Internet. Podemos verlos eligiendo en el menú principal la opción Other resources < SITES2 > (Figura 9).

Vamos a describir a continuación, brevemente, el contenido de cada uno de ellos.

- Archie es un sistema para localizar ficheros accesibles en servidores públicos en Internet. Periódicamente indiza los 2,1 millones de ficheros de programas, de datos o de texto que, aproximadamente, contienen los 1.200 servidores públicos conectados en Internet. Hytelnet 6.4 proporciona trece direcciones de estos servidores. Cuando queramos conectarnos a uno de ellos, se recomienda elegir aquel que esté más cercano geográficamente, en nuestro caso cualquiera de los tres europeos, ya que todos contienen la misma información. La obra de Ed Krol (5) explica detalladamente el funcionamiento de Archie y los procedimientos de búsqueda.

- Campus-wide information systems son sistemas de información públicos de universidades. Hytelnet recoge direcciones de cuatro países que tienen organizada esta clase de servicios accesibles en Internet: Alemania, Reino Unido, Canadá y Estados Unidos. El tipo de información que incluyen depende de la propia universidad, pero es habitual que un usuario pueda informarse de las ofertas de puestos de trabajo para profesores, política de becas, condicio- 
Figura 9

Otros recursos Internet

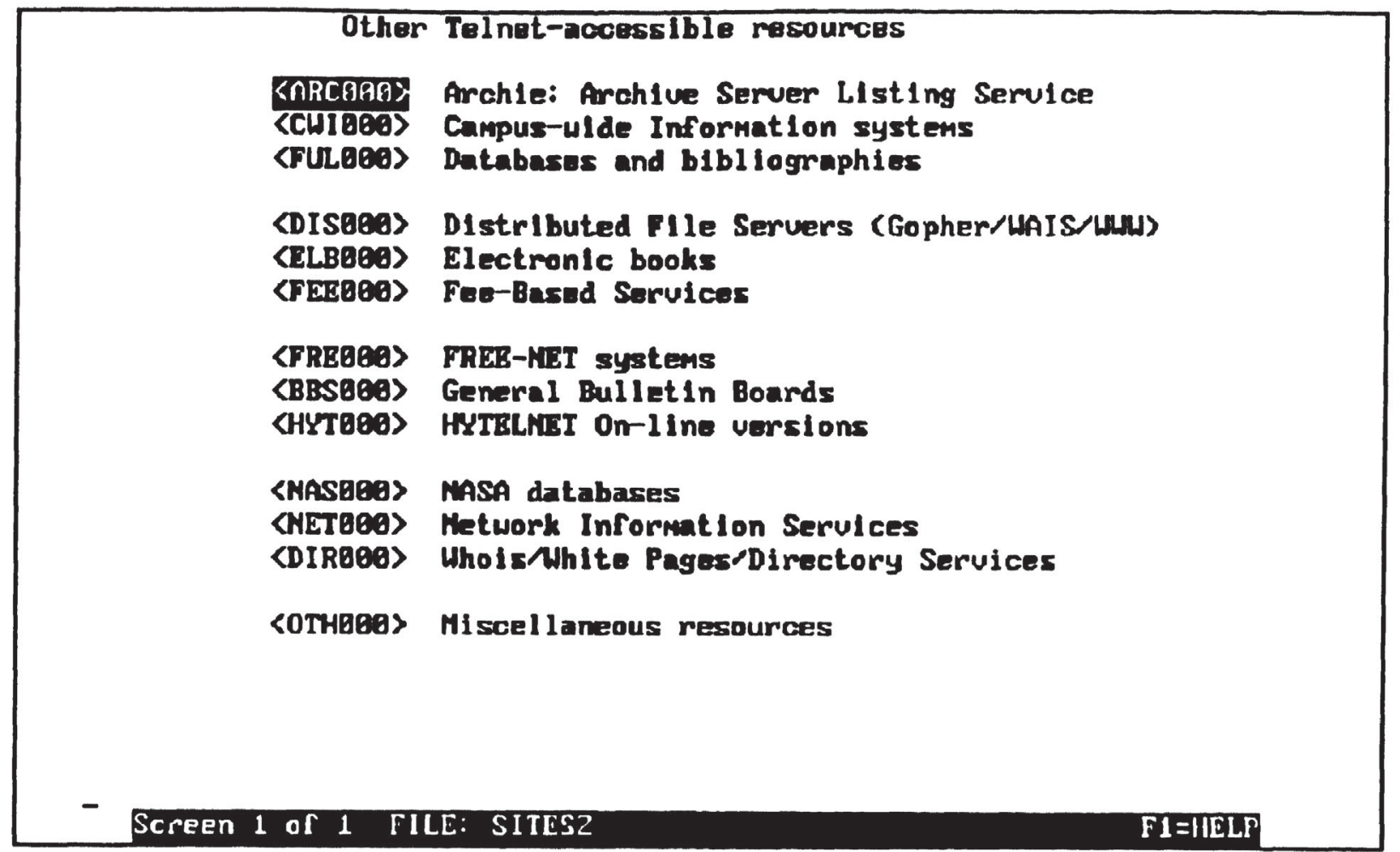

nes de matrícula, información sobre los servicios de la(s) biblioteca(s), listas de cursos y seminarios, información relativa al centro de proceso de datos, calendarios, directorios de distinta clase, guía telefónica de la propia universidad, activıdades deportivas, etc. El listado de universidades está ordenado alfabéticamente.

- Databases and bibliographies: hay una serie de bases de datos y bibliografias disponibles en la red. Desgraciadamente Hytelnet no las presenta clasificadas por temas. La versión que comentamos facilita 34 direcciones. En la mayoría de los casos se puede buscar en ellas sin limitaciones, aunque algunas requieren abrir una cuenta especial para poder acceder a su consulta (por ejemplo el British Library Document Supply Centre). Hytelnet proporciona esta información y los teléfonos de contacto.

Entre otras direcciones están las de:

- Earth Images Catalogue LEDA.

- Environmental Education Database.

- Magazine Index.

- Martin Luther King Jr. Bibliography at Stanford University.

- National Nuclear Data Center.

- ONLINE newsletter \& ONLINE libraries and microcomputers.

- ORCID: Online Retrieval of Cartographic Data.

- PINET: American Institute of Physics.

- South East Florida AIDS Information Network. 
- Distributed files servers: Gopher, WAIS y WWW son herramientas de reciente incorporación en Internet, todavía en fase de desarrollo. Permiten explorar los recursos de la red de una manera más amigable, mejorando el acceso a la información y el interfaz del usuario.

Gopher es un servidor que permite buscar fuentes de información y datos en Internet, por medio de menús, sin necesidad de saber de antemano donde reside la información. Es el propio gopher el que se ocupa de la localización y gestión de la conexión. Por ejemplo, si estamos interesados en localizar el OPAC de la Universidad Jaume I, en vez de establecer una conexión nosotros mismos, podemos optar por conectarnos a un gopher, dejar que él busque la dirección y realice la conexión. La mayor ventaja de gopher no es tanto que gestione de forma automática las conexiones Internet, sino que permita explorar fácilmente los formidables recursos de Internet. Hytelnet 6.4 lista 30 direcciones de diferentes gopher. Para usarlos cómodamente es preciso conseguir algún programa para el ordenador-cliente. Existe software de dominio público, que podemos obtener conectándonos con el gopher de la Universidad de Minnesota (5).

WAIS (Wide Area Information Systems), igual que gopher, nos permite encontrar y acceder a los recursos de la red sin necesidad de saber dónde residen. Si en gopher realizamos las búsquedas por menús, en $W A I S$ las hacemos a través de texto indizado. «Podemos realizar búsquedas por determinadas palabras o frases y el resultado serán aquellos ficheros en los que tales palabras están contenidas. Utilizando un símil, WAIS es como caminar entre las repletas estanterias de una biblioteca con una cita por única pista y obtener todos aquellos libros en los que dicha cita aparece» (6). Hytelnet 6.4 lista 8 direcciones de servidores de esta clase.

$W W W$ (World-Wide-Web) es el servicio de información más reciente de la red. Está basado en la tecnología de hipertexto. $W W W$ es un proyecto para facilitar información sobre Internet mediante documentos hipertexto. Lo desarrolla fundamentalmente el CERN, European Particle Physics Laboratory situado en Ginebra. Hytelnet 6.4 facilita la dirección de $6 W W W$.

- Electronic books, libros electrónicos. El proyecto Gutemberg es una idea ambiciosa sostenida gracias al trabajo voluntario de múltiples colaboradores. Consiste en proporcionar el mayor número de obras literarias en formato electrónico. Algunos textos ya accesibles son, por ejemplo, la Biblia, el Corán, Peter Pan, Alicia en el País de las Maravillas, las obras completas de Shakespeare, las fábulas de Esopo, tragedias de Sófocles... Hytelnet 6.4 proporciona 27 direcciones para consultar otras tantas obras.

- Fee-based services: la característica común de estos servicios es que hay que pagar una tarifa para acceder a ellos. No significa, estrictamente, que sean comerciales, ya que las tarifas varían bastante. Son muy diversos: acceso a redes de correo electrónico, servicios de búsqueda en Internet, acceso a redes de información sobre temas medioambientales, servicios de noticias especializadas (economía, música, política...), etc.

- Free-net systems, son sistemas que facilitan información local a los ciudadanos de un área geográfica determinada, normalmente a través de las bibliotecas públicas, siendo también accesibles vía Internet. Las Freenets están 
diseñadas para que los habitantes de la zona en cuestión puedan acceder a información municipal, de servicios de salud de la ciudad, a grupos de discusión, correo electrónico, revistas electrónicas y otra clase de servicios. Hytelnet incluye 20 direcciones.

- General bulletin boards, boletines electrónicos de noticias. Incluyen grupos de discusión de temas científicos y sociales (informática, bibliotecas, astrofisica, ecología, telecomunicaciones, antropología, educación...). En ocasiones se accede también a revistas especializadas en formato electrónico. Algunos de estos sitios exigen al usuario pagar una tarifa para poder usar los servicios.

- NASA databases incluye 16 direcciones de bases de datos de la NASA, accesibles en Internet.

- Newtwork information services permite, a través de Internet, conectarse a otras redes de Alemania, Holanda, Reino Unido y Estados Unidos: CONCISE, RIPE, JANET... El programa proporciona información sobre la organización y el contenido de cada una de ellas.

- Whois/white pages/directory services proporciona una relación de directorios para localizar direcciones de personas conectadas en Internet. Pueden obtenerse las direcciones de correo electrónico, teléfonos. Accediendo a los servicios de páginas blancas podemos preguntar por el nombre de una persona y obtener su dirección IP.

- Miscellaneous resources incluye servicios no clasificados en los anteriores recursos: bases de datos (ECHO, ESA), servicios de información meteorológica, servicios de pregunta/respuesta, servicios de información de la industria de ordenadores, acceso a catálogos de editoriales (Meckler), acceso a servidores con software de dominio público, servicios con información para minorías étnicas, sistemas de localización geográfica, acceso a observatorios de astronomía, servicios de búsqueda bibliográfica...

\subsection{Otros ficheros en Hytelnet}

Hay otros dos ficheros que ayudan al usuario de Internet: el Internet Glossary $<$ GLOSSARY > y el Telnet Tips < TELNET > . El primero presenta definiciones de términos de uso frecuente en la red. El segundo proporciona una serie de pistas y claves para el uso de los protocolos telnet.

\section{Direcciones telnet}

Como vemos en la Figura 6, las direcciones de conexión son las que aparecen en la primera línea:

Telnet SUN.NSF.AC.UK or 128.86.8.7.

La primera dirección (SUN.NSF.AC.UK) está escrita según el Sistema de Nombres de Dominio (Domain Name System). Es un método de administrar nombres de máquinas conectadas a Internet. Cada nivel en este sistema es denominado dominio. Los dominios están separados por puntos.

En el ejemplo (SUN.NSF.AC.UK), SUN es el nombre de un host, de un 
ordenador real. El nombre de ese ordenador lo crea y lo mantiene el grupo NSF, que debería ser el departamento donde está instalado el ordenador. El departamento NSF es una parte, dentro del grupo del Reino Unido, de centros académicos (AC). Es decir el dominio AC contiene todos los ordenadores del Reino Unido (UK) de centros académicos.

En vez de usar la primera dirección descrita, puede usarse la segunda: 128.86.8.7. Esta dirección es la conocida como dirección IP. Internet es una red de transmisión de paquetes. Las diferentes partes de Internet están conectadas entre sí por un conjunto de ordenadores denominados routers. Estas redes, en algunos casos, son Ethernets, otra son token rings, y en algunas ocasiones son redes telefónicas. Las líneas telefónicas y las Ethernets son equivalentes a los camiones y vagones postales usados por Correos. Son los medios que se usan para trasladar el correo. Los routers son equivalentes a las estafetas de Correos: toman decisiones sobre cómo dirigir el tráfico de datos, de modo similar a como las estafetas dirigen el correo postal. Cada estafeta o router no tiene una conexión directa con cada una de ellas. Si enviamos una carta de Getafe (Madrid) a Santiago de Compostela (La Coruña), Correos no reserva un tren para llevar únicamente esa carta de Madrid a La Coruña, sino que la envía a una estafeta que a su vez la envía a otra y así sucesivamente hasta que llega a su destinatario. Es decir, cada subestación sólo necesita conocer qué conexiones son posibles, y cuál es la mejor de ellas para que el sobre llegue al destinatario. De forma similar sucede en Internet: un router identifica dónde van a ir tus datos y decide dónde enviarlos. Simplemente decide cuál es el camino más lógico y lo usa. ¿Cómo sabe Internet dónde quieres comunicarte? Si se envía una carta, no basta sólo con escribirla e introducirla en un buzón. Se necesita introducirla previamente en un sobre, escribir la dirección de destino y poner el sello. Del mismo modo que Correos tiene sus reglas, Internet tiene las suyas. Las reglas se denominan protocolos. El protocolo de Internet (Internet Protocol IP) regula la sintaxis de la dirección, asegurando que los routers sepan qué hacer cuando sus datos lleguen a él. Continuando con el símil del correo, el protocolo de Internet funciona como si fuera el sobre. Esta dirección es la que permite, al igual que la comentada más arriba, que el mensaje llegue a su destino.

La dirección en Internet con la sintaxis IP consiste en cuatro números, cada uno menor de 256, que están separados por puntos, como en nuestro ejemplo del National Library of Scotland: 128.86.8.7 (7).

\section{6 ¿Cómo conseguir Hytelnet?}

La versión 6.4 del programa para DOS, está disponible vía FTP (anonymous). Para obtenerla sólo hay que seguir los siguientes pasos:
A) En el símbolo de sistema (prompt), poner: ftp access.usadk.ca.
B) En el símbolo del usuario, poner: anonymous.
C) Palabra clave (password), poner: guest o dirección del correo electrónico.
D) Símbolo del comando, poner: binary.
E) Símbolo del comando, poner: $c d$ pub/hytelnet/pc. 
F) Símbolo del comando, poner: get hytelnet NN.zip (siendo $\mathrm{NN}$ el número de la versión).

G) Símbolo del comando, poner: quit.

Se necesita el programa PKUNZIP para descomprimir Hytelnet. Existen versiones de este programa de dominio público y pueden conseguirse en la misma dirección y el mismo directorio donde está Hytelnet y que hemos descrito anteriormente. Para descomprimir el fichero con PKUNZIP puede consultarse el artículo de Rick Gates y Steve Bang (8).

Las versiones UNIX/VMS y Macintosh se obtienen en la dirección indicada anteriormente. Para más información escribir a:

- Earl Fogel a la dirección fogel@skyfox.usask.ca (para versiones UNIX/VMS).

- Charles Burchill a la direcciónburchil@ccu.umanitoba.ca. (versiones Macintosh).

Hytelnet está disponible en línea en diversos servidores de Internet. Las versiones en línea ofrecen un acceso directo a los servicios de Internet incluidos en Hytelnet. Entre ellas, destacamos:

Hytelnet de Oxford University (rsl.ox.ac.uk).

Hytelnet de University of Saskatchewan (access.usask.ca).

\section{Actualizaciones}

El programa, y ésta es una de sus mayores ventajas, se actualiza casi diariamente. Existe una lista de correo electrónico (mailing list) llamada LIB__HYTELNET, formada por más de 400 miembros de 20 países diferentes que contribuyen a su actualización. Cada dos meses aparece una nueva versión.

Si se quiere formar parte de la lista LIB_HYTELNET hay que enviar una solicitud a aa375@freenet.carleton.ca.

Si se descubre una dirección nueva en Internet o se nota algún error en alguna ya existente se puede mandar un mensaje al autor del programa a la misma dirección de correo electrónico.

Para cualquier comentario sobre el artículo pueden ponerse en contacto con:

Virginia Ortiz-Repiso, e-mailvirginia@bib.uc3m.es

Carlos Olmeda Gómez, e-mail olmeda@bib.uc3m.es.

\section{Bibliografia}

1. NATIONAL SCIENCE FOUNDATION NETWORK SERVICE CENTER. Internet resource guide. Cambridge, MA: NSF Nẹtwork Service Center, 1989. Desde abril de 1993 esta Guía está incluida en el InterNIC Directory on Database Services suministrado por AT\&T, en proceso de incorporación al InterNIC Directory of Directories (disponible via FTP, anonymous, en el host ds.internic.net, directorio resource-guide). 
2. ST. GEORGE, Art y LARSEN, Ron. Internet-Accesible library catalogs and databases. Alburquerque, NM: University of New Mexico, 1991 (puede obtenerse una copia enviando por correo electrónico el mensaje «GET LIBRARY PACKAGE» a listserv@ unmvm.bitnet).

3. BARRON, B. UNT's accessing on-line bibliographic databases. Denton, Tx: University of North Texas, 1991 (disponible vía FTP en vaxb.aes.unt.edu ó 129.120.1.4, directorio library).

4. SCOTT, P. Using Hytelnet to access Internet resources. The Public access systems review, 1992, (4), 15.

5. KROL, Ed. The whole Internet user's guide \& catalog. Sebastopol, Ca. O'Reilly \& Associates; 1992, 155-168.

6. GARCIA, F. Servicios de información y atención a usuarios de RedIRIS. Boletín de la RedIRIS, diciembre 1992, (20/21), 17-21.

7. MARTINEZ, I. Nombres y direcciones. IRIS: Boletín del Programa de Interconexion de Recursos Informáticos, 1991, (11-12), 77.

8. GATES, R. y BANG, S. Compression and archiving. The Electronic Library, 11, (2), 1993, 120-126. 